\title{
Complex Pole Placement Control for a Three-Phase Voltage Source Converter
}

\author{
F. M. Serra ${ }^{1, \dagger}$, A. Doria-Cerezo ${ }^{2}$, C. H. De Angelo ${ }^{3}$, L. L. Martín Fernández ${ }^{1}$ and M. Bodson ${ }^{4}$ \\ ${ }^{1}$ Laboratorio de Control Automático (LCA), Univ. Nac. de San Luis, Villa Mercedes, San Luis, Argentina. \\ ${ }^{2}$ Institute of Industrial and Control Engineering (IOC), Universitat Politecnica de Catalunya, Barcelona, Spain. \\ ${ }^{3}$ Inst. de Invest. en Tec. Ener. y Mat. (IITEMA), Univ. Nac. de Río Cuarto, Río Cuarto, Córdoba, Argentina. \\ ${ }^{4}$ Department of Electrical and Computer Engineering, University of Utah, Salt Lake City, USA. \\ †fmserra@unsl.edu.ar
}

\begin{abstract}
In this work, a complex-valued controller for a three-phase VSC with an LC output filter is proposed. The system is first transformed into its complex representation with the consequent order reduction and simpler analysis. The proposed complex controller places all the poles at desirable locations in the complex plane. Unlike classical controllers with an inner current-control loop and outer voltage-control loop, the proposed controller considers the complete dynamics of the system, thus ensuring the closed-loop stability. Furthermore, the complex representation facilitates the pole placement in the complex plane compared to the real domain. The performance of the proposed controller is validated through simulations and experiments.

Index Terms-complex control, LC inverter, pole placement.
\end{abstract}

\section{INTRODUCTION}

Three-phase voltage source converters (VSC) with LC output filters are widely used in applications where a sinusoidal three-phase voltage with low distortion is necessary. The most common applications of such converters include uninterruptible power supplies (UPS) [1]-[3] and DC-AC converters for isolated renewable energy generation systems [4], [5], among others.

Linear and non-linear control techniques have been applied to design controllers for VSCs with LC output filter in the aforementioned applications. Most of the schemes consist in classical approaches, such as proportional-integral (PI) controllers in the $d q$ coordinates, with two loops having different time-scales, a fast inner current-control loop and a slower outer voltage-control loop [6]. However, stability is not truly guaranteed because the complete dynamics are not considered. Usually, the design of this type of controllers includes some decoupling terms to facilitate the analysis and the tuning tasks. On the other hand, non-linear based methods tipically consider the complete dynamics, thus allowing one to perform a general stability analysis. These controllers provide satisfactory results as far as performance is concerned [2], [7], but in many cases, they can be difficult to implement and require significant computational efforts. However, a variety of tools are available for linear control design that can be exploited if the system order is low enough. In this paper, the linear approach is considered.

The mathematical model of a VSC with LC output filter consists in a sixth-order system in the original $a b c$ threephase coordinates, plus the dynamics of the load (if any).
In this paper, a three-phase VSC supplying an RL load is considered, and the complete $a b c$ model results in a ninthorder system. Order reduction is achieved using the known $a b c$ to $d q$ transformation, which results in a sixth-order system. When designing classical schemes such as PI controllers, the $d q$ axes are often decoupled by feedforward compensation, resulting in two independent dynamical systems (one for each coordinate), thus obtaining two independent systems. However, when such decoupling is used, the design flexibility is restricted.

Direct control of the output voltage is often not recommended using output feedback controllers, such as classical PI controllers. The problem comes from the fact that typical RLC values result in a pair of complex poles close to the imaginary axis that limit the closed-loop response. This problem can be solved using a controller that moves the roots to the left of the complex plane, usually through the addition of zeros. However, this scheme could add some noise problems due to high-frequency effects of the added zeros. Another option is a state-feedback scheme that allows one to place all the closedloop poles.

On the other hand, design and analysis of controllers based on transfer functions represented by complex coefficients constitute a powerful tool for three-phase electrical systems [8]. The complex-based description allows order reduction and simplification of the control analysis that results in alternative design options. This approach has recently been applied for the analysis and design of controllers in three-phase systems [9], [10] and some techniques, such as complex root locus, have been developed [11] and applied to electrical power systems and other three-phase applications [12], [13].

In this work, a control algorithm based on the complexvalued representation of the VSC feeding a resistive-inductive load, is proposed. The control scheme results in a statefeedback controller that makes it possible to freely place the closed-loop poles in the complex plane. Thanks to the complex representation, decoupling terms are intrinsically designed, resulting in a simpler analysis and greater flexibility than the traditional $d q$ representation. 


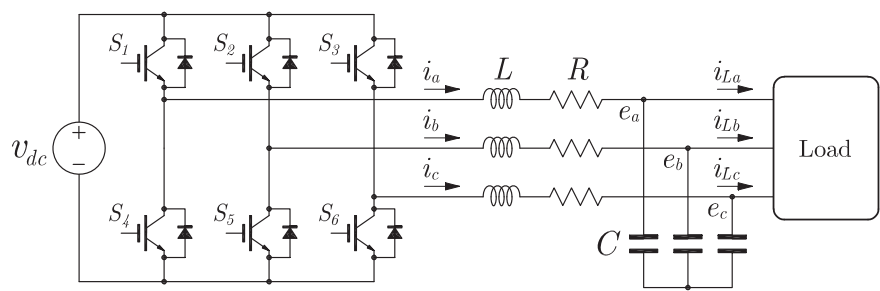

Fig. 1. Three-phase LC inverter.

\section{Dynamic Model of the VSC With LC Filter}

\section{A. Model in physical variables}

The system considered in this work is shown in Fig. 1. It is composed of a three-phase VSC with an LC output filter, where $L$ and $C$ are the inductance and capacitance of the output filter, respectively, and $R$ represents the equivalent resistance which models the total inductance losses and the converter losses. The dynamic of this converter in $a b c$ coordinates can be described by,

$$
\begin{aligned}
L \frac{d \mathbf{i}^{a b c}}{d t} & =\mathbf{M v}^{a b c}-R \mathbf{i}^{a b c}-\mathbf{M e}^{a b c}, \\
C \frac{d \mathbf{e}^{a b c}}{d t} & =\mathbf{i}^{a b c}-\mathbf{i}_{L}^{a b c},
\end{aligned}
$$

where $\mathbf{v}^{a b c}=\left[\begin{array}{lll}v_{a} & v_{b} & v_{c}\end{array}\right]^{T}$ are the output voltages of the VSC, $\mathbf{e}^{a b c}=\left[\begin{array}{lll}e_{a} & e_{b} & e_{c}\end{array}\right]^{T}$ are the voltages on the filter capacitors, $\mathbf{i}^{a b c}=\left[\begin{array}{lll}i_{a} & i_{b} & i_{c}\end{array}\right]^{T}$ are the inductor currents, $\mathbf{i}_{L}^{a b c}=\left[\begin{array}{lll}i_{L a} & i_{L b} & i_{L c}\end{array}\right]^{T}$ are the load currents, and

$$
\mathbf{M}=\frac{1}{3}\left[\begin{array}{ccc}
2 & -1 & -1 \\
-1 & 2 & -1 \\
-1 & -1 & 2
\end{array}\right]
$$

\section{B. Complex representation}

The complex representation of the system (1)-(2) is obtained by using the transformation [14],

$$
\xi=T \mathbf{x}^{a b c},
$$

where $\xi$ is a complex variable and $\mathbf{x}^{a b c}=\left[\begin{array}{lll}x_{a} & x_{b} & x_{c}\end{array}\right]^{T}$ a vector of three-phase currents or voltages. The transformation $T$ is defined as follows,

$$
T=\sqrt{\frac{2}{3}}\left[\begin{array}{lll}
1 & -\frac{1}{2}+j \frac{\sqrt{3}}{2} & -\frac{1}{2}-j \frac{\sqrt{3}}{2}
\end{array}\right] e^{-j \theta},
$$

where $\theta$ is the instantaneous phase of a reference signal, and $\frac{\mathrm{d} \theta}{\mathrm{d} t}=\omega$ is its frequency. Then, the $d q$ representation of threephase variables can be obtained from,

$$
\xi=x_{d}+j x_{q}
$$

where $x_{d}$ is the real part and $x_{q}$ is the imaginary part of the complex variable. Note that the homopolar component is zero because the three-phase VSC with LC output filter is a three wire system.
Using (3) in (1)-(2), the $d q$ state-space model is obtained, which results in the following complex system,

$$
\begin{aligned}
L \frac{d i}{d t} & =v-(R+j \omega L) i-e, \\
C \frac{d e}{d t} & =i-j \omega C e-i_{L},
\end{aligned}
$$

where $i=i_{d}+j i_{q}, e=e_{d}+j e_{q}, v=v_{d}+j v_{q}$ and $i_{L}=$ $i_{L d}+j i_{L q}$ are complex variables.

The model defined by (6) and (7) is for an arbitrary load. With the complex representation, the resulting system is a second order system, while the original system was of order six in $a b c$ coordinates, and order four if the classical $d q$ representation were used.

A resistive-inductive (RL) load is considered in this work. The state-space equations for the VSC with an LC filter and RL load are given by,

$$
\begin{aligned}
L \frac{d i}{d t} & =v-(R+j \omega L) i-e, \\
C \frac{d e}{d t} & =i-j \omega C e-i_{L}, \\
L_{L} \frac{d i_{L}}{d t} & =e-\left(R_{L}+j \omega L_{L}\right) i_{L},
\end{aligned}
$$

where $L_{L}$ and $R_{L}$ are the inductance and resistance of the load respectively. The inclusion of the load model increases the system order to three. The open-loop dynamics can be written as,

$$
\left[\begin{array}{ccc}
N_{L}(s) & 1 & 0 \\
-1 & N_{C}(s) & 1 \\
0 & -1 & N_{L L}(s)
\end{array}\right]\left[\begin{array}{c}
i \\
e \\
i_{L}
\end{array}\right]=\left[\begin{array}{l}
v \\
0 \\
0
\end{array}\right]
$$

where,

$$
\begin{aligned}
N_{L}(s) & =(s+j \omega) L+R, \\
N_{C}(s) & =(s+j \omega) C, \\
N_{L L}(s) & =(s+j \omega) L_{L}+R_{L} .
\end{aligned}
$$

The voltage of the VSC is $v=m v_{d c}$, where $m=m_{d}+j m_{q}$ is the modulation index.

The response from the modulation index to the load voltage is,

$$
e=\frac{v_{d c} N_{L L}(s)}{D_{O L}(s)} m
$$

where,

$$
\begin{aligned}
D_{O L}(s) & =N_{L}(s)+N_{L}(s) N_{C}(s) N_{L L}(s)+N_{L L} \\
& =N_{R}(s)+j N_{I}(s),
\end{aligned}
$$

$N_{R}(s)$ is the real part and $N_{I}(s)$ is the imaginary part of $D_{O L}(s)$, that is

$$
\begin{aligned}
N_{R}(s) & =L L_{L} C s^{3}+C\left(L R_{L}+L_{L} R\right) s^{2} \\
& +\left(L+L_{L}+R R_{L} C-3 \omega^{2} L L_{L} C\right) s \\
& +\left(R+R_{L}-\omega^{2} C\left(L R_{L}+L_{L} R\right)\right) \\
N_{I}(s) & =3 \omega L L_{L} C s^{2}+2 \omega C\left(L R_{L}+L_{L} R\right) s \\
& -\omega^{3} L L_{L} C+\omega R R_{L} C+\omega\left(L+L_{L}\right) .
\end{aligned}
$$




$$
\begin{aligned}
& D_{C L}(s)=L L_{L} C s^{4}+\left(C\left(L R_{L}+L_{L} R\right)+k_{L} v_{d c} L_{L} C\right) s^{3} \\
+ & \left(L+L_{L}-3 \omega^{2} L L_{L} C+R R_{L} C+k_{L} v_{d c} R_{L} C+k_{P} v_{d c} L_{L}+j 2 \omega k_{L} v_{d c} L_{L} C\right) s^{2} \\
+\quad & \left(R+R_{L}-\omega^{2} C\left(L R_{L}+L_{L} R\right)+k_{L} v_{d c}-\omega^{2} k_{L} v_{d c} L_{L} C+k_{P} v_{d c} R_{L}+k_{I} v_{d c} L_{L}+k_{L L} v_{d c}\right. \\
+\quad & \left.j\left(\omega k_{L} v_{d c} R_{L} C+\omega k_{P} v_{d c} L_{L}\right)\right) s+k_{I} v_{d c} R_{L}+j \omega k_{I} v_{d c} L_{L} .
\end{aligned}
$$

$$
\begin{aligned}
k_{I}= & \frac{L L_{L} C\left(p_{1} p_{2} p_{3} p_{4}\right)}{v_{d c}\left(R_{L}+j \omega L_{L}\right)}, \\
k_{L}= & -\frac{L L_{L} C\left(p_{1}+p_{2}+p_{3}+p_{4}\right)-C\left(L R_{L}+L_{L} R\right)}{L_{L} C v_{d c}}, \\
k_{P}= & \frac{L L_{L} C\left(p_{1} p_{2}+p_{1} p_{3}+p_{1} p_{4}+p_{2} p_{3}+p_{2} p_{4}+p_{3} p_{4}\right)+L+L_{L}-3 \omega^{2} L L_{L} C+k_{L} v_{d c} R_{L} C+j 2 \omega k_{L} v_{d c} L_{L} C}{L_{L} v_{d c}}, \\
k_{L L}= & -\frac{L L_{L} C\left(p_{1} p_{2} p_{3}+p_{1} p_{2} p_{4}+p_{1} p_{3} p_{4}+p_{2} p_{3} p_{4}\right)}{v_{d c}} \\
& -\frac{\left(R+R_{L}-\omega^{2} C\left(L R_{L}+L_{L} R\right)+k_{L} v_{d c}-\omega^{2} k_{L} v_{d c} L_{L} C+k_{I} v_{d c} L_{L}+k_{P} v_{d c} R_{L}+j\left(\omega k_{L} v_{d c} R_{L} C+\omega k_{P} v_{d c} L_{L}\right)\right)}{v_{d c}} .
\end{aligned}
$$

\section{Complex Pole Placement Control}

Similarly to the control schemes presented in [12] and [13] the following controller is proposed,

$m=j \frac{N_{I}(s)}{v_{d c} N_{L L}(s)} e-k_{L} i-k_{L L} i_{L}+k_{P}\left(1+\frac{1}{T_{i} s}\right)\left(e^{\mathrm{ref}}-e\right)$,

that includes the feedback of the inductor and load current. The values of the controller parameters $\left(k_{L}, k_{L L}, k_{P}\right.$ and $\left.T_{i}\right)$ will be specified using a pole placement method.

Substituting the proposed controller (19) in (15), the closedloop dynamics are determined by,

$$
e=\frac{v_{d c} N_{L L}(s)\left(k_{P} s+k_{I}\right)}{D_{C L}(s)} e^{\mathrm{ref}},
$$

where,

$$
\begin{aligned}
D_{C L}(s) & =N_{R}(s) s+k_{L} v_{d c}\left(1+N_{C}(s) N_{L L}(s)\right) s \\
& +k_{L L} v_{d c} s+k_{P} v_{d c} N_{L L}(s)\left(s+\frac{1}{T_{i}}\right) .
\end{aligned}
$$

By using (17) and (13) in (21), $D_{C L}(s)$ results as shown in (22).

The control parameters in (19) $\left(k_{L}, k_{L L}, k_{P}\right.$ and $\left.T_{i}=\frac{k_{P}}{k_{I}}\right)$ are obtained by matching the fourth-order system in (22) with the desired characteristic equation, $D_{C L}^{d}(s)$, given by,

$$
\begin{aligned}
D_{C L}^{d}(s)= & \left(s-p_{1}\right)\left(s-p_{2}\right)\left(s-p_{3}\right)\left(s-p_{4}\right) \\
= & s^{4}-\left(p_{1}+p_{2}+p_{3}+p_{4}\right) s^{3} \\
& +\left(p_{1} p_{2}+p_{1} p_{3}+p_{1} p_{4}+p_{2} p_{3}+p_{2} p_{4}+p_{3} p_{4}\right) s^{2} \\
& -\left(p_{1} p_{2} p_{3}+p_{1} p_{2} p_{4}+p_{1} p_{3} p_{4}+p_{2} p_{3} p_{4}\right) s \\
& +p_{1} p_{2} p_{3} p_{4},
\end{aligned}
$$

resulting in the controller gains shown in (24), (25), (26) and (27).
Remark: the complex controller in (19) is equivalent to a real controller in the $d q$ representation. However, the controller would not naturally be obtained with a conventional $d q$ design. When designing in the $d q$ framework, one is usually decoupling $d$ and $q$ dynamics in order to facilitate the analysis as two separate SISO systems. However, when including the feedforward term, the dynamics become coupled again and the analysis requires MIMO tools. The complex design is a SISO design that intrinsically controls the corresponding, coupled $2 \times 2$ MIMO system in the real domain.

\section{A. Control gains determination}

The controller parameters, (24), (25), (26) and (27) are determined through desired closed-loop poles $p_{1}, \ldots, p_{4}$, which are themselves obtained from specifications in the timedomain. The roots of the characteristic equation are expressed as follows,

$$
p_{i}=-\zeta_{i} \omega_{n_{i}} \pm j \omega_{n_{i}} \sqrt{1-\zeta_{i}^{2}}=-\sigma_{i} \pm j \omega_{i},
$$

with,

$$
\sigma_{i}=\zeta_{i} \omega_{n_{i}}
$$

where the subscript $i$ is indicative of each pole of the system.

Using the criterion of $2 \%$ for the settling time of the dominant pole, $p_{1}$,

$$
t_{s_{1}}=\frac{4}{\sigma_{1}}
$$

where $\sigma_{1}$ can be calculated for a desired time.

In the application considered in this work, the settling time should be around one cycle of the voltage waveform, i.e. $t_{s_{1}}=$ $0.02 \mathrm{~s}$, which results in $\sigma_{1}=200$. A design choice made in this paper is to set the pole $p_{1}$ according to this rule, and move the remaining three poles to locations whose real parts are at 
TABLE I

SELECTED Poles.

\begin{tabular}{cc}
\hline Pole & Value \\
\hline$p_{1}$ & $-200+j 0$ \\
$p_{2}$ & $-2500+j 5000$ \\
$p_{3}$ & $-2500-j 7200$ \\
$p_{4}$ & $-14000-j 100$ \\
\hline
\end{tabular}

TABLE II

SYSTEM PARAMETERS.

\begin{tabular}{cc}
\hline Parameter & Value \\
\hline$L$ & $2.25 \mathrm{mH}$ \\
$R$ & $0.2 \Omega$ \\
$C$ & $45 \mathrm{uF}$ \\
$L_{L}$ & $3.45 \mathrm{mH}$ \\
$R_{L}$ & $50 \Omega$ \\
$v_{d c}$ & $300 \mathrm{~V}$ \\
$\omega$ & $314.16 \mathrm{rad} / \mathrm{s}$ \\
\hline
\end{tabular}

least $5 \sigma_{1}$ or $10 \sigma_{1}$. In this manner, the pole $p_{1}$ is dominant. The imaginary parts of the poles are chosen such that,

$$
-\frac{\operatorname{Re}\left(p_{i}\right)}{\left|p_{i}\right|}<5 .
$$

The poles for the system, selected according to the above criteria, are presented in Table I.

Using the selected poles and the system parameters shown in Table II, the gains of the controllers can be determined and result in the values shown in Table III.

\section{Simulation AND EXPERIMENTAL RESUlts}

In order to evaluate the performance of the complex controller proposed in this paper, simulation and experimental tests were performed.

\section{A. Simulation results}

Simulation results were obtained using SimPowerSystem from Matlab. The simulated converter includes switching effects, losses and detailed transistor models.

Fig. 2 shows a test where a change in the output voltage reference is produced at $0.1 \mathrm{~s}$, and a load change occurs at 0.2 s. Fig. 2(a) shows the $d q$ components of the output voltage. As can be observed, when a change of reference is made at $0.1 \mathrm{~s}$, the $d$ axis component changes from 50 to 100 in $20 \mathrm{~ms}$, with a minimum overshoot. The $q$ axis component remains at zero value, as expected for a real reference value $e^{\text {ref }}$. At $0.2 \mathrm{~s}$, a load change occurs where $R_{L}$ changes from $50 \Omega$ to

TABLE III

CONTROLler PARAMETERS.

\begin{tabular}{cc}
\hline Pole & value \\
\hline$k_{P}$ & $0.0111+j 3.4589 \times 10^{-4}$ \\
$k_{I}$ & $2.7593+j 0.3185$ \\
$k_{L}$ & $0.0346+j 0.0172$ \\
$k_{L L}$ & $-0.040+j 0.0312$ \\
\hline
\end{tabular}
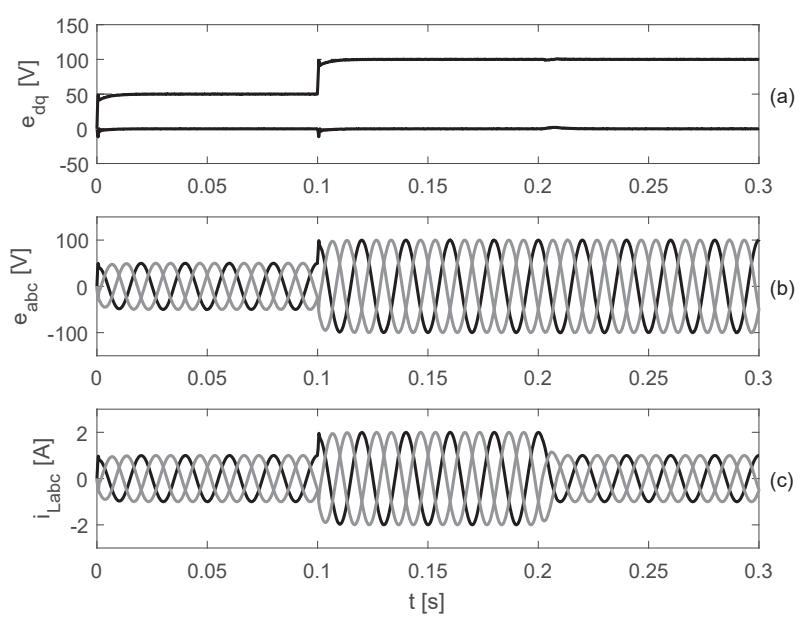

Fig. 2. Performance of the proposed controller for RL load. (a) $d q$ voltage components, (b) three-phase output voltage in $a b c$ coordinates, (c) three-phase output current in $a b c$ coordinates.

$100 \Omega$ with $L_{L}=4.5 \mathrm{mH}$. It can be observed that the $d q$ components of the output voltage remain at their references values. It is worth noticing that under load changes closedloop poles move. For a load of $100 \Omega$ the closed-loop poles are $p_{1}=-199.79-j 6.8046, p_{2}=-2231.2+j 4938.3$, $p_{3}=-2498.8-j 7237.3$ and $p_{4}=-28763+j 5.8206$, and the closed-loop system remains stable and exhibits a similar performance.

The output voltage in $a b c$ coordinates is shown in Fig. 2(b) while the inductor current in the same coordinates is presented in Fig. 2(c). It can be observed that the output voltage remains sinusoidal, with the required amplitude and frequency.

\section{B. Experimental results}

The experimental tests were performed using a laboratory prototype consisting of a three-phase VSC with an LC output filter. The parameters used in the experimental tests are the same as used during the simulation stage (Table II). The inverter has a Semix-101GD12E4s module of Semikron $(1200 \mathrm{~V}, 100 \mathrm{~A})$ and the control algorithm was implemented in a TMS320F28335 floating point DSP of Texas Instruments.

To avoid the need for a second current sensor for the load, an option was considered to let the gain $k_{L L}=0$. Using the control gains obtained in the previous section, see Table III except for $k_{L L}=0$, the closed-loop poles were found to move to $p_{1}=-189.4-j 9.672, p_{2}=-2261.4+j 5143.1$, $p_{3}=-2529.5-j 7456.9$ and $p_{4}=-14220+j 23.448$. With the change, the dominance of $p_{1}$ over $p_{2}, p_{3}, p_{4}$ remains, and the response time only increases by $5 \%$. See in Fig. 3, the original closed-loop poles and the new values with $K_{L L}=0$. This option was implemented in the experiments.

Fig. 4 shows the generated three-phase output voltage when the reference is $e^{\text {ref }}=100+j 0 \mathrm{~V}$ and the desired frequency is set at $50 \mathrm{~Hz}$. The waveform is sinusoidal, while the amplitude and frequency remain in their reference values. Fig. 5 shows the three-phase current when a resistive-inductive load of $50 \Omega$ and $3.45 \mathrm{mH}$ is considered. 


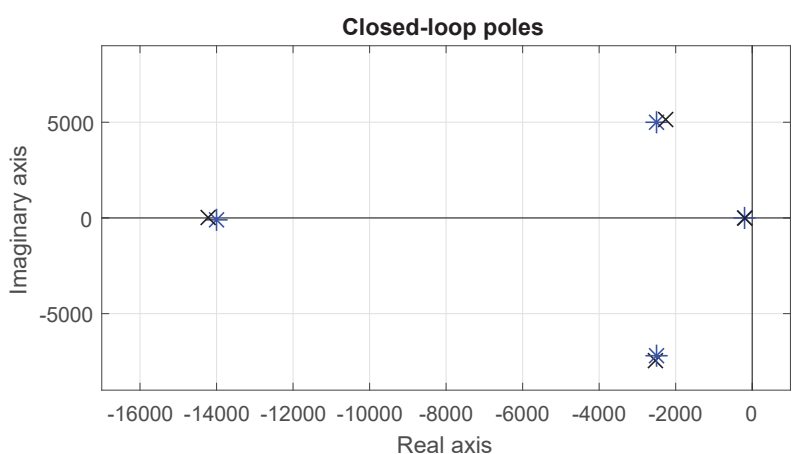

Fig. 3. Comparison between the closed-loop poles for $k_{L L}=-0.040+$ $j 0.0312$ (blue) and $k_{L L}=0$ (black).

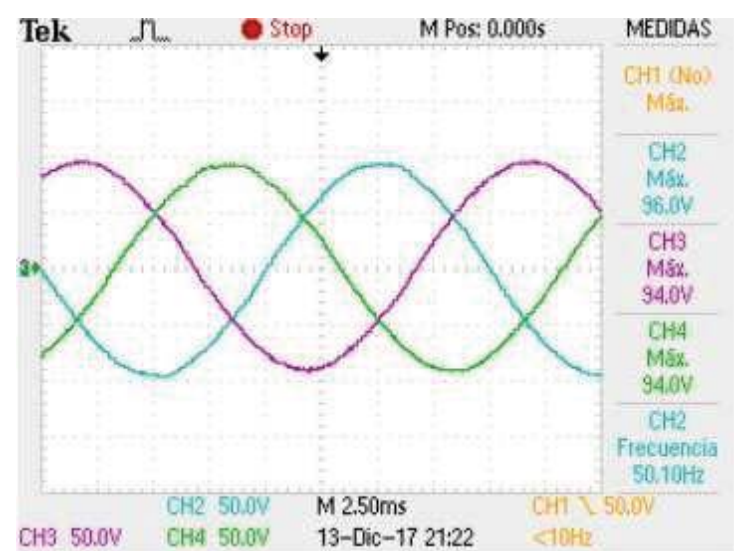

Fig. 4. Three-phase output voltage.

A reference change in the amplitude of the three-phase output voltage from $50 \mathrm{~V}$ to $100 \mathrm{~V}$ is shown in Fig. 6 (CH1). The amplitude of the voltage $(\mathrm{CH} 2$ to $\mathrm{CH} 4)$ follows the reference value and the new value is reached in less than one cycle. Fig. 7 shows the three-phase currents $(\mathrm{CH} 2$ to $\mathrm{CH} 4)$ next to the voltage of phase $a(\mathrm{CH} 1)$ for this test.

The $d q$ components of the voltages for this test are shown in Fig. 8. When a change of reference is made, the $d$ axis

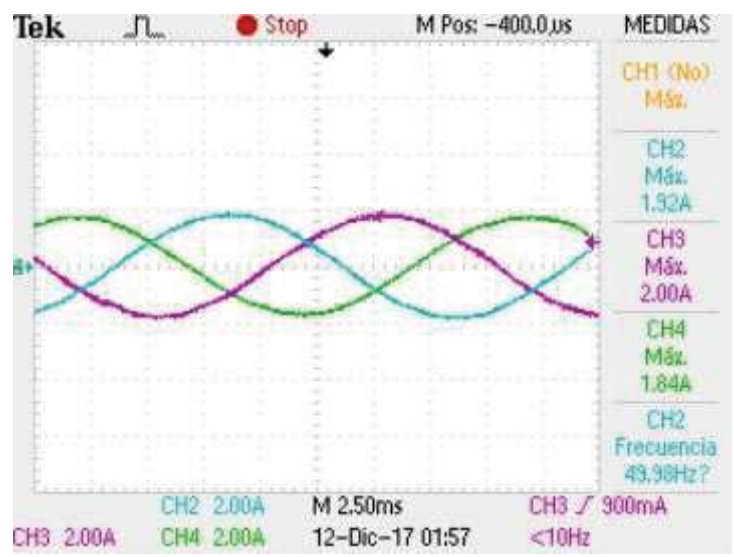

Fig. 5. Three-phase current for an RL load.

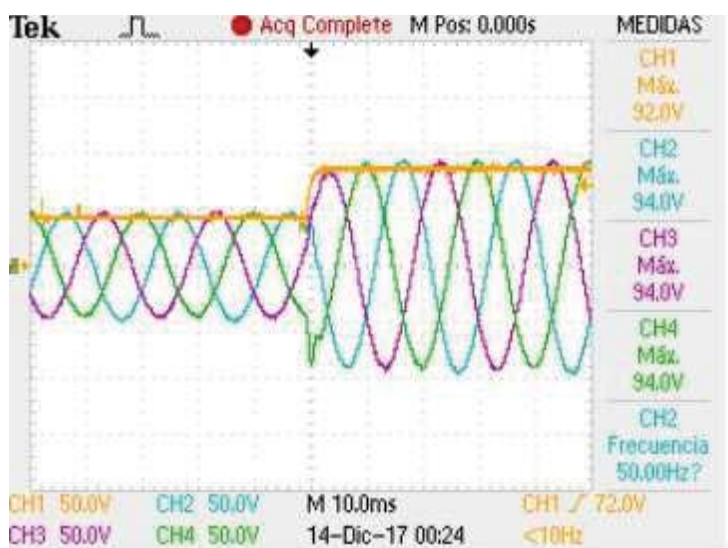

Fig. 6. Three-phase output voltage for a reference change. CH1: Reference. $\mathrm{CH} 2$ to $\mathrm{CH} 4$ : Output voltages.

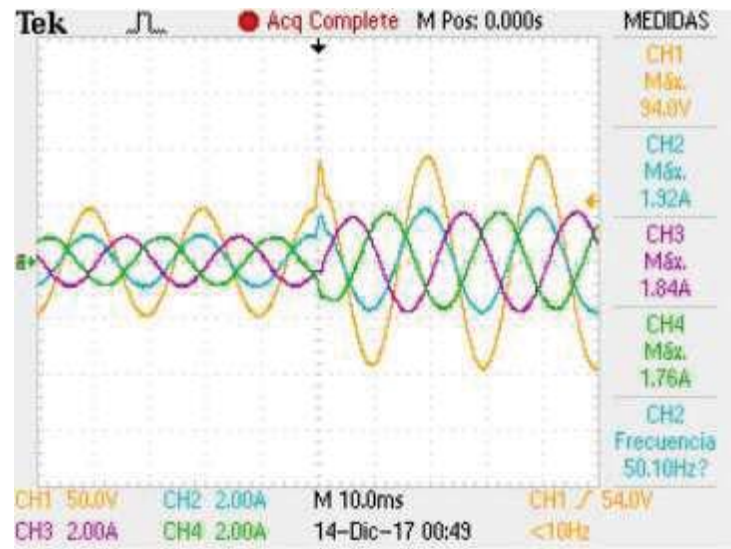

Fig. 7. Three-phase current $(\mathrm{CH} 2$ to $\mathrm{CH} 4)$ and voltage of phase $a(\mathrm{CH} 1)$ for a reference change.

component changes from $50 \mathrm{~V}$ to $100 \mathrm{~V}$ in $20 \mathrm{~ms}$, with a minimum overshoot. As expected, the $q$ axis component remains at zero.

The performance of the proposed controller when a load change is applied is shown in Fig 9. The same test as in the simulation case was performed where $R_{L}$ changes from $50 \Omega$ to $100 \Omega$ and $L_{L}$ remains in $3.45 \mathrm{mH}$. Fig. 9 shows the threephase output voltages $(\mathrm{CH} 2$ to $\mathrm{CH} 4)$ and the current of phase $a(\mathrm{CH} 1)$. The amplitude and frequency of the output voltage

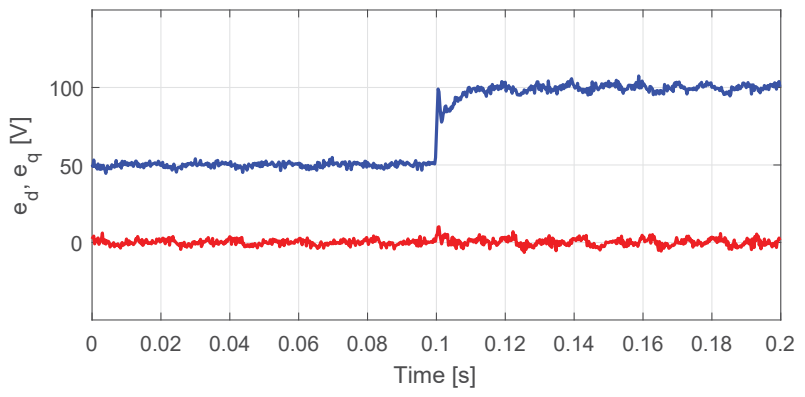

Fig. 8. $d$ (blue) and $q$ (red) component voltages for a reference change. 


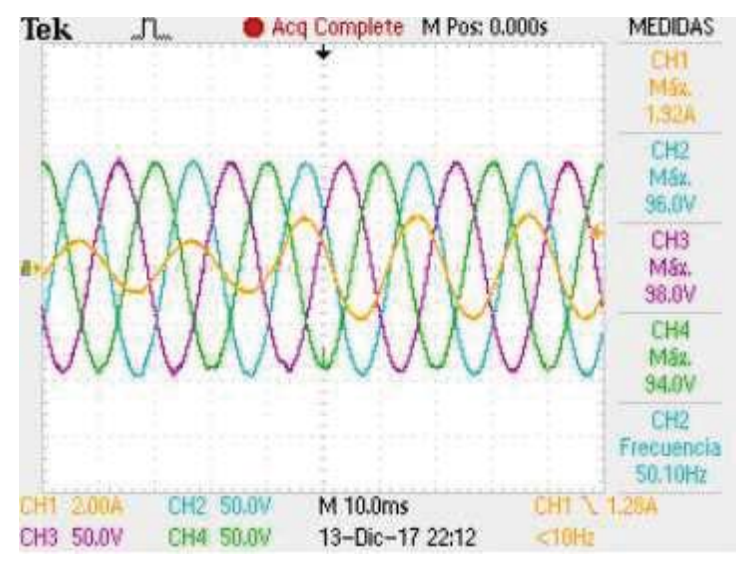

Fig. 9. Three-phase output voltages $(\mathrm{CH} 2$ to $\mathrm{CH} 4)$ and current of phase $a$ (CH1) for a load change.

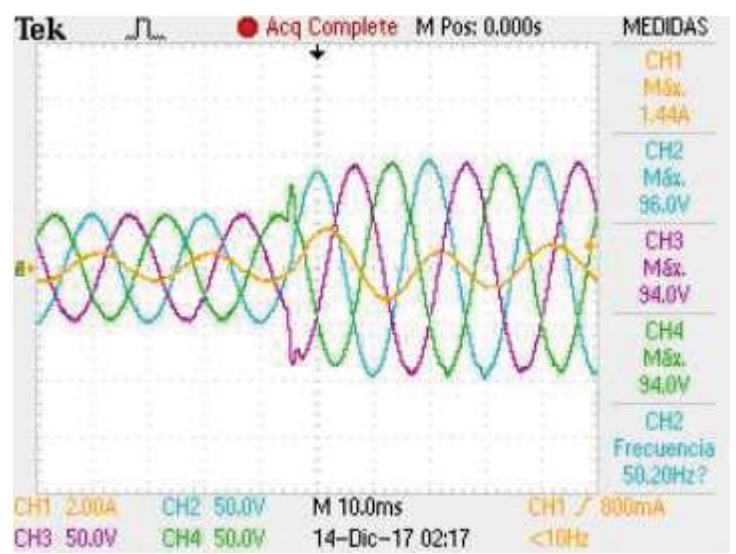

Fig. 10. Three-phase output voltages $(\mathrm{CH} 2$ to $\mathrm{CH} 4)$ and current of phase $a$ (CH1) when an induction machine is connected to the system.

do not present visible deviations, while the amplitude of the current increases to the new value required by the load.

In Fig. 10, the performance of the proposed controller is shown when a induction machine is connected to the system, and the reference of the output voltage is changed from $50 \mathrm{~V}$ to $100 \mathrm{~V}$ at $0.45 \mathrm{~s}$.

\section{CONCLUSIONS}

The use of the complex-valued representation of a threephase power converter facilitates the synthesis and design of controllers. The system order is reduced and simpler solutions can be found without the explicit use of decoupling terms. Thanks to these features, the performance of the closed-loop system can be improved. The proposed structure allows the user to place the closed-loop poles at desired values ensuring a response in the time domain as specified by a dominant pole.

\section{ACKNOWLEDGEMENTS}

The work of F. M. Serra, C. H. De Angelo and L. L Martín Fernández was supported by the Universidad Nacional de San Luis; Universidad Nacional de Río Cuarto; FONCyT; ANPCyT; and the Consejo Nacional de Investigaciones Científi cas y Técnicas (CONICET).
The work of A. Dòria-Cerezo was supported in part by the Government of Spain through the Agencia Estatal de Investigación Project DPI2017-85404-P and in part by the Generalitat de Catalunya through the Project 2017 SGR 872.

\section{REFERENCES}

[1] G. Escobar, A. A. Valdez, J. Leyva-Ramos and P. Mattavelli. "Repetitive-Based Controller for a UPS Inverter to Compensate Unbalance and Harmonic Distortion," IEEE Transactions on Industrial Electronics. Vol. 54, No. 1, pp. 504-510, 2007. DOI. 10.1109/TIE.2006.888803.

[2] D. E. Kim and D. C. Lee. "Feedback Linearization Control of Three-Phase UPS Inverter Systems," IEEE Transactions on Industrial Electronics. Vol. 57, No. 3, pp. 963-968, 2010. DOI. 10.1109/TIE.2009.2038404.

[3] Zhang Yu, Li Minying and Kang Yong. "PID Controller Design for UPS Three-Phase Inverters Considering Magnetic Coupling" Energies. Vol. 7, No. 12, pp. 8036-8055, 2014. DOI. 10.3390/en7128036.

[4] T. D. Do, V. Q. Leu, Y. S. Choi, H. H. Choi and J. W. Jung "An Adaptive Voltage Control Strategy of Three-Phase Inverter for Stand-Alone Distributed Generation Systems," IEEE Transactions on Industrial Electronics. Vol. 60, No. 12, pp. 5660-5672, 2013. DOI. 10.1109/TIE.2012.2230603

[5] D. Q. Dang, Y. S. Choi, H. H. Choi and J. W. Jung. "Experimental Validation of a Fuzzy Adaptive Voltage Controller for Three-Phase PWM Inverter of a Standalone DG Unit," IEEE Transactions on Industrial Informatics. Vol. 11, No. 3, pp. 632-641, 2015. DOI. 10.1109/TII.2015.2416981.

[6] M. E. Haque, M. Negnevitsky and K. M. Muttaqi. "A Novel Control Strategy for a Variable-Speed Wind Turbine With a Permanent-Magnet Synchronous Generator," IEEE Transactions on Industry Applications. Vol. 46, No. 1, pp. 331-339, 2010. DOI. 10.1109/TIA.2009.2036550.

[7] F. M. Serra, C. H. De Angelo and D. G. Forchetti. "IDA-PBC control of a DCAC converter for sinusoidal three-phase voltage generation,' International Journal of Electronics. Vol. 104, No. 1, pp. 93-110, 2017. DOI. 10.1080/00207217.2016.1191087.

[8] L. Harnefors, "Modeling of three-phase dynamic systems using complex transfer functions and transfer matrices," IEEE Transactions on Industrial Electronics. Vol. 54, No. 4, pp. 2239-2248, 2007. DOI. 10.1109/TIE.2007.894769.

[9] M. Bodson. "Design of controllers in the complex domain," 53rd IEEE Conference on Decision and Control. pp. 4077-4082, 2014, Los Angeles, CA. DOI. 10.1109/CDC.2014.7040023.

[10] M. Bodson and O. Kiselychnyk. "The complex Hurwitz test for the analysis of spontaneous self-excitation in induction generators," IEEE Transactions on Automatic Control. Vol. 58, No. 2, pp. 449-454, 2013 DOI. 10.1109/TAC.2012.2206691.

[11] A. Dòria-Cerezo and M. Bodson. "Root locus rules for polynomials with complex coefficients," 21st Mediterranean Conference on Control and Automation. pp. 663-670, 2013, Chania. DOI. 10.1109/MED.2013.6608794.

[12] A. Dòria-Cerezo and M. Bodson. "Design of Controllers for Electrical Power Systems Using a Complex Root Locus Method," IEEE Transactions on Industrial Electronics. Vol. 63, No. 6, pp. 3706-3716, 2016 DOI. 10.1109/TIE.2016.2521599.

[13] A. Dòria-Cerezo, F. M. Serra and M. Bodson, "Complex-Based Controller for a Three-Phase Inverter With an LCL Filter Connected to Unbalanced Grids," IEEE Transactions on Power Electronics. Vol. 34 No. 4, pp. 3899-3909, 2019. DOI. 10.1109/TPEL.2018.2854576.

[14] H. J. Baesmat and M. Bodson, "Suppression of Sub-Synchronous Resonances through Excitation Control of Doubly-Fed Induction Generators," IEEE Transactions on Power Systems. Vol. 58, No. 2, pp. 449-454, 2013. DOI. 10.1109/TPWRS.2019.2922406. 